\title{
OPTIMIZATION OF CERTAIN COMPONENTS OF QUADRILATERALS: A SIMPLIFIED APPROACH TO OLD AND NEW PROBLEMS
}

\author{
MOSHE STUPEL ${ }^{1,2}$, AVI SIGLER ${ }^{2}$ and JAY JAHANGIRI ${ }^{3,4}$ \\ ${ }^{1}$ Gordon Academic College of Education \\ Haifa \\ Israel \\ e-mail: stupel@bezeqint.net \\ 2Shannan Academic College of Education \\ Haifa \\ Israel \\ e-mail: avibsigler@gmail.com \\ ${ }^{3}$ Mathematical Sciences \\ Kent State University \\ Kent, Ohio \\ USA \\ ${ }^{4}$ Mathematical Sciences \\ Kent State University Geauga and Twinsburg \\ Regional Academic Center \\ Burton, Ohio \\ USA \\ e-mail: jjahangi@kent.edu
}




\begin{abstract}
The optimization of components of quadrilaterals, such as area and product of diagonals, can be obtained using calculus optimization. Without resorting to calculus, we use simple algebraic and geometrical identities and provide simplified approaches to these problems and many more. Such mathematical activity can lead to a deeper study of the discovery of hidden extreme properties in different geometrical figures.
\end{abstract}

\title{
1. Introduction
}

The problem of the maximal area of a convex polygon with a given perimeter is known as the isoperimetric problem and was solved in the 19th century: the circle has the maximal area (e.g., see Andreescu et al. [1]). Some 1800 years ago the Greek Mathematician Pappus proved that of all $n$-sided polygons with a given perimeter, the perfect polygon is the one with the largest area (e.g., see Thomas [2,3]). It is customary to use calculus in a variety of optimization problems including geometry. It is the aim of this article to use only arithmetic and geometrical facts and ideas to solve certain old and new optimization problems concerning the components of quadrilaterals.

In the sequel (unless otherwise stated), we consider only convex polygons and let $\check{s}$ represent the semi-perimeter and $\breve{A}$ represent the area of any given polygon.

The following area formula was (independently) discovered by the German mathematicians Carl Anton Bretschneider and Karl Georg Christian von Staudt in 1842 (e.g., see Johnson [4]).

Theorem 1.1 (Bretschneider-Staudt). The area of an arbitrary convex quadrilateral $A B C D$ with side lengths are $a, b, c$, and d (e.g., see Figure 1) is given by $\breve{A}$, where

$$
\breve{A}=\sqrt{(\breve{s}-a)(\breve{s}-b)(\breve{s}-c)(\breve{s}-d)-a b c d \cdot \cos ^{2} \frac{\angle A+\angle C}{2}} .
$$


The following corollaries are the obvious consequence of Theorem1.1.

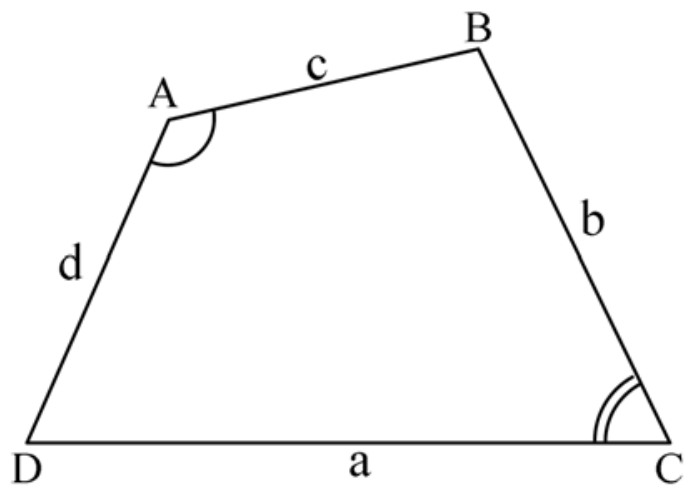

Figure 1.

Corollary 1.2. If a circle can be inscribed in the given quadrilateral $A B C D$, then we have $\check{s}-d=b, \breve{s}-b=d, \breve{s}-c=a$, and $\check{s}-a=c$. Therefore the area $\breve{A}$ of the quadrilateral $A B C D$ is given by

$$
\breve{A}=\sqrt{a b c d} \cdot \sin \frac{\angle A+\angle C}{2} .
$$

Corollary 1.3. If the quadrilateral $A B C D$ is circumscribable, i.e., if it's opposite interior angles are supplementary, then the area $\breve{A}$ of the quadrilateral reduces to Brahmagupta's formula (e.g., see Hess [5])

$$
\breve{A}=\sqrt{(\breve{s}-a)(\breve{s}-b)(\breve{s}-c)(\breve{s}-d)} .
$$

If we let $d=0$, i.e., if we chose a triangle instead of a quadrilateral, then the Corollary 1.3 reduces to the Heron's triangle formula (also see Hess [5]).

Corollary 1.4. If the quadrilateral $A B C D$ is circumscribable as well as a circle can be inscribed in $A B C D$, then the area $\breve{A}$ of the quadrilateral $A B C D$ is given by $\breve{A}=\sqrt{a b c d}$. 
Corollary 1.5. According to the Bretschneider-Staudt theorem, the area of a quadrilateral is maximized if the quadrilateral is circumscribable $\left(\angle A+\angle C=180^{\circ}\right)$.

\section{Area Maximization}

Now we are equipped to state and prove the following four theorems on the maximal area of convex quadrilaterals with a given perimeter and different degrees of freedom. First we deal with the case of given perimeter and no other restrictions.

Theorem 2.1. The maximal area of a quadrilateral with fixed perimeter $2 \check{s}$ is $\frac{\breve{s}^{2}}{4}$, that is, the quadrilateral is a square.

Proof. First we note that the maximum area $\breve{A}$ occurs if $A B C D$ is circumscribable. So, we have

$$
\breve{A} \leq \sqrt{(\breve{s}-a)(\breve{s}-b)(\breve{s}-c)(\breve{s}-d)} .
$$

Now, the proof is obtained by using the relationship between the arithmetic and geometric means (e.g., see the article by the first author [6], p. 936):

$$
\sqrt[4]{(\breve{s}-a)(\breve{s}-b)(\breve{s}-c)(\breve{s}-d)} \leq \frac{(\breve{s}-a)+(\breve{s}-b)+(\breve{s}-c)+(\breve{s}-d)}{4}=\frac{\breve{s}}{2} .
$$

It easily follows that the right hand side of the above inequality is maximized if $a=b=c=d$ which is exactly the area of a square with $\operatorname{sides} \frac{\breve{s}}{2}$.

The next theorem is when the perimeter and two sides of a quadrilateral are fixed.

Theorem 2.2. The maximal area of a quadrilateral with fixed perimeter $2 \check{s}$ and given sides $a$ and $b$ is $\breve{A}_{\max }=\frac{a+b}{2} \cdot \sqrt{(\breve{s}-a)(\breve{s}-b)}$. 
Proof. By the hypothesis, $\breve{s}-a$ and $\breve{s}-b$ are given and fixed. Therefore, using the relation between the mathematical averages yields the inequality

$$
(\breve{s}-c)(\breve{s}-d) \leq\left(\frac{2 \breve{s}-c-d}{2}\right)^{2}=\left(\frac{a+b}{2}\right)^{2} .
$$

Since the maximum area occurs if $A B C D$ is circumscribable, the above inequality in conjunction with Theorem 1.1 yield $\breve{A} \leq \sqrt{(\breve{s}-a)(\breve{s}-b)\left(\frac{a+b}{2}\right)^{2}}$. This completes the proof.

The following remark is worth noticing.

Remark 2.3. (i) If $a$ and $b$ are the opposite sides, then the maximum area occurs for $A B C D$ being an isosceles trapezoid.

(ii) If $a$ and $b$ are the adjacent sides, then $\breve{s}-\frac{a+b}{2}=c=d$.

Next we deal with a case of the above theorem if only one side is fixed.

Theorem 2.4. The maximal area of a quadrilateral with fixed perimeter $2 \check{s}$ and only one side a (say) given is $\breve{A} \leq \sqrt{(\breve{s}-a)\left(\frac{\breve{s}+a}{3}\right)^{3}}$.

Proof. Since the perimeter $2 \breve{s}$ and the side $a$ are given, $\breve{s}-a$ is fixed. Now, using the relation between the mathematical averages, we obtain

$$
(\breve{s}-b)(\breve{s}-c)(\breve{s}-d) \leq\left(\frac{3 \breve{s}-b-c-d}{3}\right)^{3}=\left[\frac{3 \breve{s}-(2 \breve{s}-a)}{3}\right]^{3}=\left(\frac{\breve{s}+a}{3}\right)^{3} .
$$

Since the maximum area $\breve{A}$ occurs if $A B C D$ is circumscribable, it follows from Theorem 1.1 and the above inequality that $\breve{A} \leq \sqrt{(\breve{s}-a)\left(\frac{\breve{s}+a}{3}\right)^{3}}$. This completes the proof. 
Remark 2.5. In the case of Theorem 2.4, a simple algebra reveals that $A B C D$ is an isosceles trapezoid with one side $a$ and the other three sides equal to $\frac{2 \breve{s}-a}{3}$.

In the next theorem, we discuss the area of a quadrilateral whose sides are consecutive natural numbers.

Theorem 2.6. If the sides of a circumscribable quadrilateral are consecutive natural numbers, its area cannot be a natural number.

Proof. For the natural number $n$, let the sides of the circumscribable quadrilateral be $a=n, b=n+1, c=n+2, d=n+3$, and $\check{s}$ be half its perimeter. Then the area of the quadrilateral is $\breve{A}=$ $\sqrt{(\breve{s}-a)(\breve{s}-b)(\breve{s}-c)(\breve{s}-d)}$.

Substituting for $a, b, c, d$, and $\check{s}$ we obtain $\breve{A}=\sqrt{n(n+1)(n+2)(n+3)}$. Squaring both sides and adding 1 yields

$$
\begin{aligned}
\breve{A}^{2}+1 & =n(n+1)(n+2)(n+3)+1 \\
& =\left(n^{2}+3 n\right)\left(n^{2}+3 n+2\right)+1 \\
& =\left(n^{2}+3 n\right)^{2}+2\left(n^{2}+3 n\right)+1 \\
& =\left(n^{2}+3 n+1\right)^{2} .
\end{aligned}
$$

This means that $\breve{A}^{2}=\left(n^{2}+3 n+1\right)^{2}-1$ and therefore $\breve{A}$ cannot be a natural number.

\section{Diagonals of Quadrilaterals}

In this section, we discuss a variety of cases involving the product of diagonals of a given quadrilateral.

First we give the necessary condition for maximizing the product of diagonals of a given quadrilateral. 
Theorem 3.1. The maximal product of diagonals of a quadrilateral occurs if no two largest or no two smallest sides are adjacent.

Proof. Let $a, b, c$, and $d$ denote the sides of the given quadrilateral. Without loss of generality, we assume that $a \geq b \geq c \geq d$. Then there are the following three possible arrangements of the sides of this quadrilateral (Figures 2.I, 2.II, 3.III).
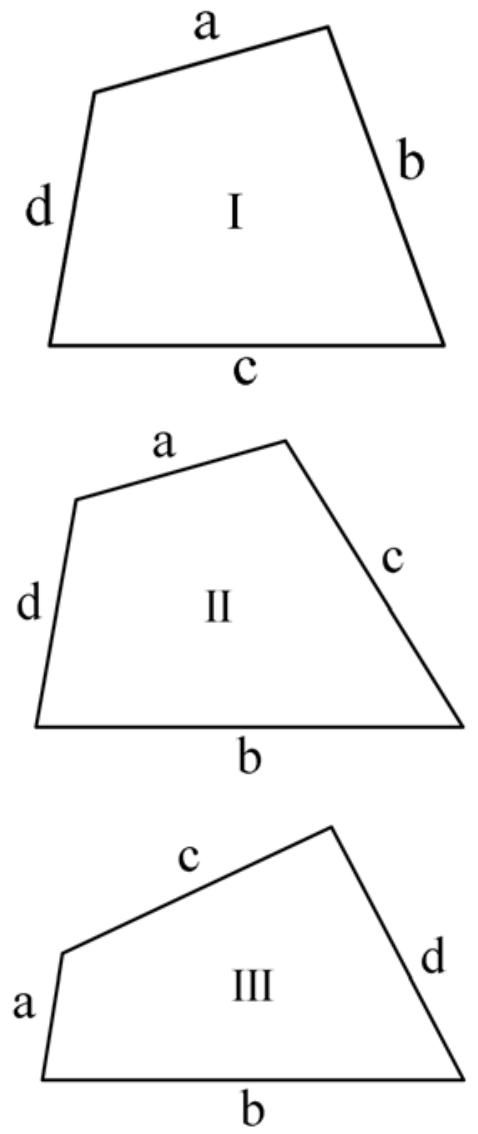

Figure 2. 
Denote the diagonals of the quadrilateral by $p$ and $q$. Applying Ptolemy's Theorem (e.g., see [7] or [8]) to Figures 2.I, 2.II, and 2.III, we, respectively, obtain

$$
\begin{cases}\text { I } & b d+a c \geq p q, \\ \text { II } & a b+c d \geq p q, \\ \text { III } & b c+a d \geq p q .\end{cases}
$$

We note that

$$
(a b+c d)-(b d+a c)=b(a-d)-c(a-d)=\underbrace{(b-c)}_{\geq 0} \underbrace{(a-d)}_{\geq 0} \geq 0
$$

and

$$
(a b+c d)-(b c+a d)=b(a-c)-d(a-c)=\underbrace{(b-d)}_{\geq 0} \underbrace{(a-c)}_{\geq 0} \geq 0
$$

Therefore, $a b+c d>b d+a c$ and $a b+c d>b d+a c$. This completes the proof. We note that the equality in Theorem 3.1 occurs if the quadrilateral is inscribable.

In the following theorem, we determine the actual upper bound for the products of the diagonals of a given quadrilateral.

Theorem 3.2. Consider the quadrilateral $A B C D$ with given sides $a, b$, $c, d$ and diagonals $A C$ and $B D$ (Figure 3$)$. Then,

$$
A B^{2}+C D^{2}+2 \cdot A D \cdot B C \geq A C^{2}+B D^{2} .
$$




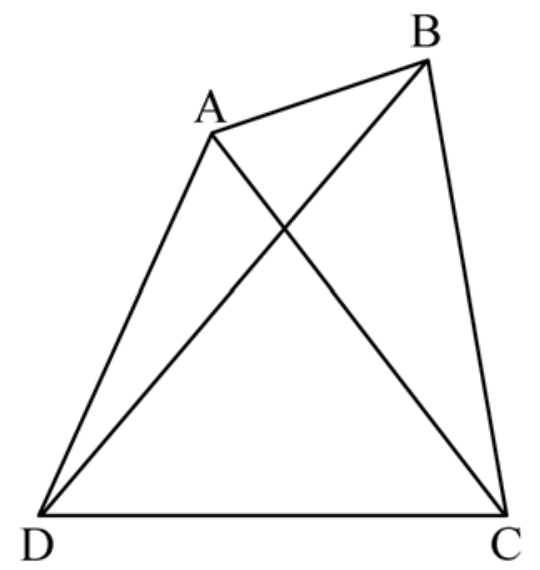

Figure 3.

In order to prove the above theorem, we shall need the following wellknown Euler's Theorem (e.g., see Pinasco [9]).

Euler's Theorem 3.3 ([9]). In the quadrilateral $A B C D$ let the points $M$ and $N$ be the midpoints of the diagonals $B D$ and $A C$, respectively (Figure 4). Then,

$$
A B^{2}+B C^{2}+C D^{2}+A D^{2}=A C^{2}+B D^{2}+4 M N^{2} .
$$

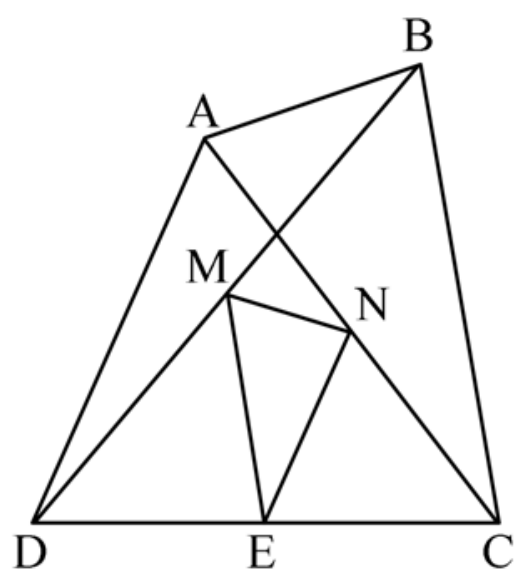

Figure 4. 
Proof of Theorem 3.2. Let the point $E$ be the middle of the side $D C$ (Figure 4). Clearly, $N E=\frac{1}{2} A D$ and $M E=\frac{1}{2} B C$.

It is also clear that the length of a side in a triangle is larger than the difference of the other two sides, and therefore $M N \geq|M E-N E|$ or $M N \geq\left|\frac{B C-A D}{2}\right|$. This in conjunction with Euler's Theorem 3.3 yields

$$
A B^{2}+B C^{2}+C D^{2}+A D^{2} \geq A C^{2}+B D^{2}-2 \cdot B C \cdot A D,
$$

or

$$
A B^{2}+B C^{2}+2 \cdot B C \cdot A D \geq A C^{2}+B D^{2} .
$$

We note that the equality holds if $M N=\left|\frac{B C-A D}{2}\right|$. In the other words, $B C \| A D$ which means that the maximal value of $A C^{2}+B D^{2}$ is obtained when $A B C D$ is a trapezoid. The following corollary can easily be verified using Theorems 3.1 and 3.2.

Corollary 3.4. Consider the quadrilateral $A B C D$ with given sides a, $b$, $c$, and $d$ and diagonals $A C$ and $B D$. Then the maximal value of $A C^{2}+B D^{2}$ occurs if $A B$ and $C D$ are the two largest non-adjacent sides of the quadrilateral $A B C D$.

Next we regard the diagonals of a quadrilateral as vectors and discuss their scalar product.

Theorem 3.5. Consider the quadrilateral ABCD whose side lengths are $a, b, c, d$ (Figure 5). Then the scalar product $|\overrightarrow{C A} \cdot \overrightarrow{D B}|$ is constant and is equal to $\left|\frac{\left(a^{2}+c^{2}\right)-\left(b^{2}+d^{2}\right)}{2}\right|$. 


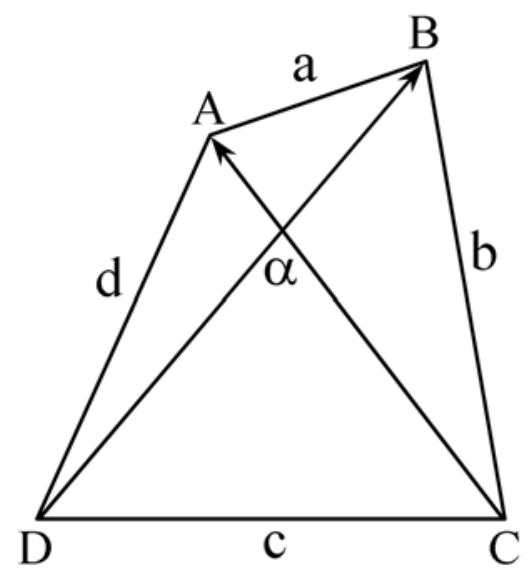

Figure 5.

Proof. From the generalized law of cosines (e.g., see Collins and Osler [10]) applied to the quadrilateral $A B C D$, we obtain

$$
\left(d^{2}+b^{2}\right)-\left(a^{2}+c^{2}\right)=2 A C \cdot B D \cdot \cos \alpha .
$$

On the other hand, we have $\overrightarrow{A C} \cdot \overrightarrow{B D} \equiv|A C| \cdot|B D| \cdot \cos \alpha$. This completes the proof of the theorem.

Substituting $\quad a=a, b=a+d, c=a+3 d, \quad$ and $\quad d=a+2 d \quad$ in Theorem 3.5 yields the following corollary.

Corollary 3.6. Consider the quadrilateral ABCD (Figure 6) whose side lengths constitute an arithmetic sequence. Then $|\overrightarrow{A C \cdot B D}|=2 d^{2}$. 


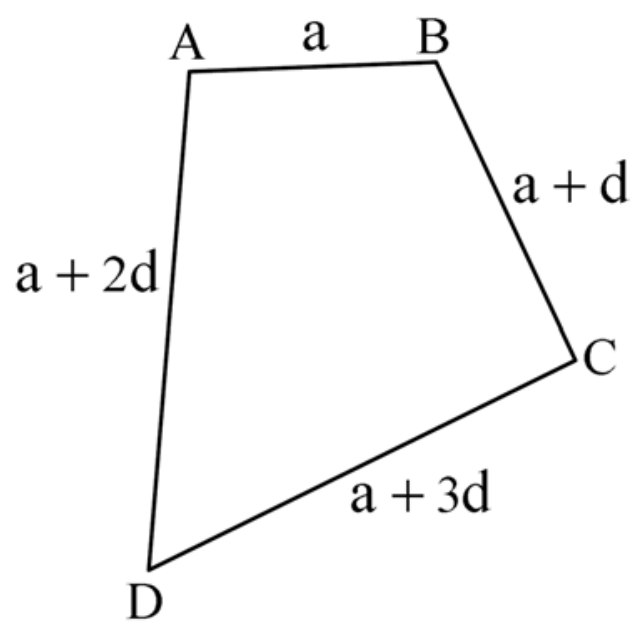

Figure 6.

The following corollary also follows from Theorem 3.5.

Corollary 3.7. Consider the quadrilateral ABCD (Figure 7) so that, $a+c=b+d$.

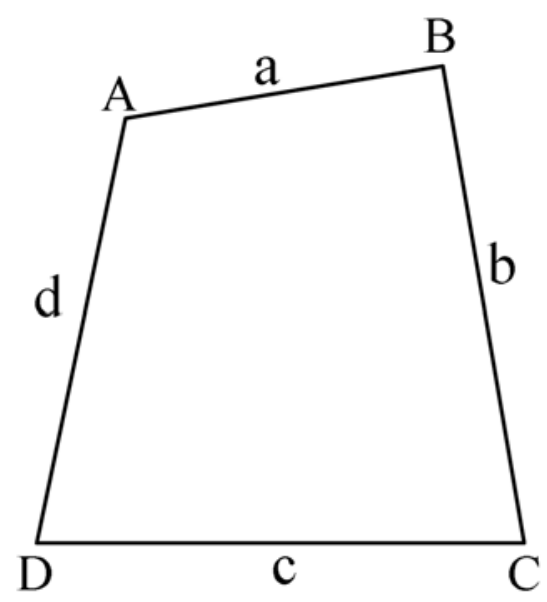

Figure 7. 
If each side of the quadrilateral $A B C D$ is increased by a constant $m$ (Figure 8), then we have $\overrightarrow{A_{1} C_{1}} \cdot \overrightarrow{B_{1} D_{1}}=\overrightarrow{A C} \cdot \overrightarrow{B D}$.

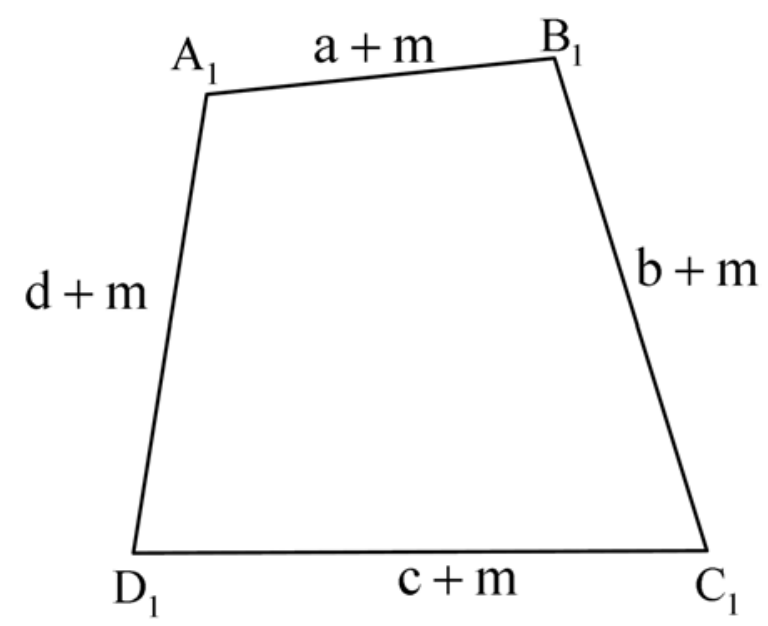

Figure 8.

This can be easily justified, since substituting $a=a+m, b=b+m$, $c=c+m$, and $d=d+m$ in Theorem 3.5 and using a simple algebra reveals that

$$
\left(a^{2}+c^{2}\right)-\left(b^{2}+d^{2}\right)=(a+m)^{2}+(c+m)^{2}-\left[(b+m)^{2}+(d+m)^{2}\right] .
$$

\section{Summary}

Calculus techniques are indeed the principal tools for solving optimization problems in various fields of mathematics. The present paper presents optimizations of components of convex quadrilaterals where extrema are found using other alternative methods, such as algebraic and geometrical methods. It is not the intension of this article to downplay the importance of the use of calculus techniques in optimization but to explore other alternative venues. The use of alternative methods highlights mathematics as a magical field with surprising solutions. Also, and more importantly, our approach is straightforward, student friendly, and can easily be used in school and college level geometry classes. 


\section{References}

[1] T. Andreescu, O. Mushkarov and L. Stoyanov, Geometric Problems on Maxima and Minima, Birkhauser, Boston, Inc., Boston, MA, 2006. ISBN: 978-0-8176-3517-6.

[2] I. B. Thomas, Selections Illustrating the History of Greek Mathematics, Vol. I, Forgotten Books, February 8, 2018. ISBN-13: 978-0656101535.

[3] I. B. Thomas, Selections Illustrating the History of Greek Mathematics, Vol. II, Forgotten Books, July 25, 2017. ISBN-13: 978-0282549244.

[4] R. A. Johnson, Advanced Euclidean Geometry, Dover Publication, 2007. ISBN13:978-0486462370.

[5] A. Hess, A highway from Heron to Brahmagupta, Forum Geometricorum 12 (2012), 191-192. MR2955633.

[6] R. Mogilevsky and M. Stupel, Using the wonder of inequalities between averages for mathematics problems solving, Educational Research and Reviews 11(9) (2016), 932-944.

DOI: https://doi.org/10.5897/ERR2016.2763

[7] J. Haantjes, Ptolemy's theorem, Simon Stevin 29 (1952), 25-31. MR0048827.

[8] R. Askey, Completing Brahmagupta's Extension of Ptolemy's Theorem, The Legacy of Alladi Ramakrishnan in the Mathematical Sciences, Springer, New York, 2010, pp. 191-197. ISBN-13: 978-1441962621.

DOI: https://doi.org/10.1007/978-1-4419-6263-8_11

[9] J. P. Pinasco, New proofs of Euclid's and Euler's theorems, American Mathematical Monthly 116(2) (2009), 172-174. MR2478061.

[10] L. Collins and T. J. Osler, Law of cosines generalized for any polygon and any polyhedron, The Mathematical Gazette 95(533) (2011), 240-243.

DOI: https://doi.org/10.1017/S0025557200002953 